\title{
Elastic light scattering from free sub-micron particles in the soft X-ray regime
}

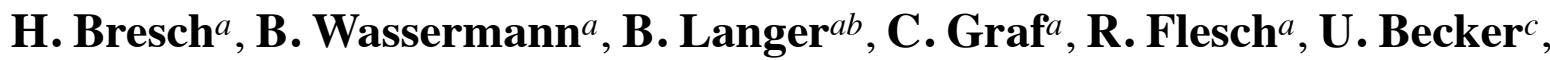 \\ B. Österreicher ${ }^{d}$, T. Leisner ${ }^{d e}$ and E. Rühl ${ }^{* a}$
}

aPhysikalische und Theoretische Chemie, Institut für Chemie und Biochemie, Freie

Universität Berlin, Takustr. 3, 14195, Berlin, Germany. E-mail:

ruehl@chemie.fu-berlin.de; Fax: +49 308385 2717; Tel: +49 3083852396

bMax-Born-Institut, Max-Born-Str. 2a, 12489, Berlin, Germany

cFritz-Haber-Institut der MPG, Faradayweg 4-6, 14195, Berlin, Germany

dFakultät für Mathematik und Naturwissenschaften, TU Ilmenau, Max-Planck-Ring 14, 98693, Ilmenau, Germany

eIMK, Forschungszentrum Karlsruhe, Postfach 3640, 76021, Karlsruhe, Germany

We report the first experimental results on angle-resolved elastic light scattering in the soft X-ray regime, where free sub-micron particles in the size regime between 150 and 250 $\mathrm{nm}$ are studied in the gas phase by using a continuous particle beam. Two different types of studies are reported: (i) Angle-resolved elastic light scattering experiments provide specific information on the scattering patterns in the regime of element-selective inner-shell excitation near the Si 2p-edge (80-150 eV). In addition to intense forward scattering, we observe distinct features in the angle-resolved scattering patterns. These are modelled by using Mie theory as well as a model that includes contributions from diffuse and specular reflection. The results are primarily attributed to scattering from soft X-rays in the surface layer. (ii) Spectroscopic experiments are reported, where the photon detector is placed at a given scattering angle while scanning the photon energy near the Si 2p-absorption edge. These results are also analyzed by a Mie model, yielding accurate information of the size distribution.

\section{Introduction}

Elastic light scattering from free particles is an approach that has been successfully used for many years to probe the size, shape, and index of refraction of microparticles.[14] Pioneering work goes back to Gustav Mie a century ago, who reported the assignment of light scattering patterns of colloidal metal particles.[5]

It is well-known that Mie scattering is accompanied by a typical intense forwardscattering lobe for $x \gg 1$, where $x$ is the Mie size parameter $(x=\pi D / \lambda)$. Here, $D$ corresponds to the diameter (size) of the particle and $\lambda$ is the wavelength of the incident radiation. Moreover, distinct variations in the scattered light intensity give a unique signature of the particle's optical properties. These unique features allow one in the UV and visible regime to derive, via Mie simulations, the particle size and the index of refraction with high accuracy.[2,6] 
More recent work has focused on short wavelength radiation in Vacuum UltraViolet (VUV), as a promising way to study sub-micron particles.[7] It has been shown that sizes of free particles below $D \approx 300 \mathrm{~nm}$ can only be probed by VUV-radiation, whereas ultraviolet and visible light are suitable to probe optical properties of microparticles by elastic light scattering. Note, that ensembles of nanoparticles in liquid dispersions can be properly sized by visible light. The difference compared to the present work is that light scattering from particles in solution relies on fluctuations of the refractive index, which are caused by fluctuations in density and concentration.[8] Thus, the scattered light signal may be influenced by other factors, such as interactions of the particles with the solvent. Note also, that size information on single nanoparticles in the gas phase via elastic light scattering cannot be derived in the visible regime. This is because with visible light, nanoparticles have Mie size parameters around or well below unity. In this regime, the scattering cross section drops rapidly with decreasing $x$ and scales as $R^{6} / \lambda^{4}$, where $R$ is the radius of the particle. Additionally, the angle-resolved scattering patterns become similar to that of Hertz dipole in the regime of $x \leq 1$.

Angle-resolved light scattering patterns of free nanoparticles have been measured in a continuous particle beam approach, which is similar to the present experimental setup. $[7,9,10]$ Short wavelength radiation in the VUV and soft X-ray regimes has the inherent advantage that the Mie size parameter of sub-micron particles becomes sufficiently large with increasing photon energy to observe properties of Mie scattering. However, besides intense forward scattering, previous work has shown little evidence of Mie resonances so far. $[7,9,10]$ This is mostly due to changes in the index of refraction, particle size distributions in the beam, and a somewhat limited range of accessible scattering angles in previous measurements (cf. ref. $[7,9,10])$.

Evidence that elastic light scattering from single, size-selected particles has been observed, arising from an alternative approach for plotting the experimental results.[11] Angle-resolved light scattering can alternatively be presented by plotting the dimensionless parameter $q R$, where $q$ is the scattering wave vector, instead of the scattering angle.[1113] This approach yields, besides evidence for Mie resonances, slopes of -2 or -4 , which have been assigned in terms of the phase shift, multiple scattering, and the illuminated portion of the particles. Indeed, previous work in the VUV regime clearly indicated that such even-numbered slopes occur.[9]

Besides free particles in the gas phase, numerous works have been performed in which deposited samples of particulate matter were studied in the soft X-ray regime.[14] XRays are known to be suitable to probe structured surfaces by scattering experiments.15 Specifically, soft X-ray scattering from deposited nanoparticles of polymers has been studied recently, where the region of small values of $q$ was investigated, yielding accurate size information.[16]

In general, it is expected that investigations of free nanoparticles in the gas phase are advantageous compared to studies on the corresponding adsorbed species, since there is no influence of any substrate. Moreover, multiple scattering between closely spaced particles (cf. ref. [17]) is inhibited, which permits the application of Mie formalism for data analysis.

We report in this study first experimental results on angle-resolved elastic light scattering in the soft X-ray regime, where free sub-micron particles are studied in the continuous beam. This approach is complementary to single trapped nanoparticles, where the charging mechanisms were derived recently.[18] However, in trapped, single particle experi- 
ments, radiation damage may occur, which can change the particle properties.[19] The present approach overcomes this inherent drawback, since a constantly fresh sample of well defined, size-selected, sub-micron particles is studied. We use for the present work silica nanoparticles in the size regime between $150 \mathrm{~nm}$ and $250 \mathrm{~nm}$ as a simple and robust model system. Related to the present work are soft X-ray reflectivity measurements on amorphous $\mathrm{SiO}_{2}$, which yield near-edge spectra and the optical constants in the $\mathrm{Si}$ 2p-regime.[20-24]

\section{Experimental}

Free variable size nanoparticles are prepared in a continuous beam, similar to previous work.[7,9,10] This approach makes use of the following components, which are described in more detail in the following: (i) sample preparation by generating and spraying a dispersion of nanoparticles into a controlled gas phase at ambient pressure by using an atomizer (TSI 3076); (ii) size selection is accomplished by an electrostatic classifier, yielding isolated nanoparticles of defined size (TSI 3080L); (iii) transfer of the particles into the high vacuum chamber and focusing of the continuous particle beam by an aerodynamic lens system, including a triple differential pumping system; (iv) interaction with a beam of monochromatic X-rays from the storage ring BESSY (Berlin, Germany); (v) angle-resolved detection of the scattered radiation.

Variable size silica nanoparticles are prepared by chemical syntheses,[9] which rely on the Stöber approach.[25,26] The particles are repeatedly cleaned by centrifugation and redispersion in purified ethanol. The particle sizes (and standard deviations) are determined by Transmission Electron Microscopy (TEM), yielding: (i) $147 \mathrm{~nm}( \pm 4.8 \%)$, (ii) $188 \mathrm{~nm}$ $( \pm 4.2 \%)$, and (iii) $251 \mathrm{~nm}( \pm 3.4 \%)$. Subsequently, the particle sizes (and relative widths of the size distributions at full width half maximum) are measured by the particle sizer: (i) 151 $\mathrm{nm}( \pm 10.0 \%)$, (ii) $202 \mathrm{~nm}( \pm 7.4 \%)$ and (iii) $250 \mathrm{~nm}( \pm 6.0 \%)$. These sizes are determined by a calibrated Differential Mobility Analyzer (DMA), which is equipped by a condensation particle counter (particle sizer system TSI 3936L22). They are fully consistent with those from TEM, where the increased widths of the distributions in DMA-sizing are primarily due to the resolution of this device. The widths of the particle size distributions are expected to decrease with particle size,[9] as observed in TEM and DMA measurements. Considering the particle sizes and their size distributions, as determined by two independent approaches, we refer to these sizes by rounded values in the following discussion. These correspond to $\sim 150 \mathrm{~nm}, \sim 200 \mathrm{~nm}$, and $\sim 250 \mathrm{~nm}$, respectively. We use these rounded values throughout this work.

The liquid dispersions containing the nanoparticles of well-defined size are diluted to $\sim 0.5 \mathrm{~g} / \mathrm{L}^{-1}$. Recent work has shown that high particle concentrations in the liquid phase lead to the enhanced formation of aggregates after they are transferred into the gas phase by spraying the liquid samples into the atmosphere at ambient pressure.[9] The droplets are dried in a diffusion dryer (TSI 3062) and neutralized by a ${ }^{85} \mathrm{Kr}$ source (TSI 3077). This yields a beam of isolated silica nanoparticles of well-defined size. The particles are either singly or doubly charged, where the charge state is inferred from the particle sizer (TSI 3936L22), as shown in Fig. 1. The particle sizer consists of a differential mobility analyzer (TSI 3081) and a condensation particle counter (TSI 3022A). As a result of the control of particle size in the gas phase, the particle beam has been used in this study without primary size selection. This provides a substantial increase in target density. Typical particle densities of $\geq 6 \times 10^{6}$ particles per $\mathrm{cm}^{3}$ are obtained from this particle preparation, which is sufficient for angle-resolved light scattering experiments. The particles are focused by an 
aerodynamic lens system. It is mounted behind a primary aperture of $180 \mu \mathrm{m}$, so that the pressure at its entrance is reduced from ambient pressure to $\sim 10 \mathrm{mbar}$. The lens system has a total length of $300 \mathrm{~mm}$. It consists of six apertures, which are mounted at equal distances of $50 \mathrm{~mm}$ with orifices decreasing in downstream direction from $5.3 \mathrm{~mm}$ to $3.9 \mathrm{~mm}$. We note that this design is similar to that published earlier in ref. 27 . The present lens system yields an optimum transmission range between $70 \mathrm{~nm}$ and $300 \mathrm{~nm}$, where the maximum of particles is detected at sizes of $200 \mathrm{~nm}$ at an air flow of $0.37 \mathrm{~L} \mathrm{~min}^{-1}$. The pressure at the exit of the aerodynamic lens is typically of the order of $0.1 \mathrm{mbar}$. The focused aerosol beam is transferred into the scattering chamber via a triple differential pumping stage.

The scattering chamber is kept under high vacuum during operation. The base pressure in the scattering chamber is $\sim 10^{-8} \mathrm{mbar}$, it increases to $\sim 3 \times 10^{-7} \mathrm{mbar}$ during the experiments. The particle beam has in the scattering chamber a diameter of $\leq 500 \mu \mathrm{m}$. It is crossed in the middle of this chamber by monochromatic synchrotron radiation from the UE49/2-PGM1 and UE52-SGM beam lines at the storage ring facility BESSY (Berlin, Germany). The typical energy resolving power $(E / \Delta E)$ of these undulator beam lines is ap-

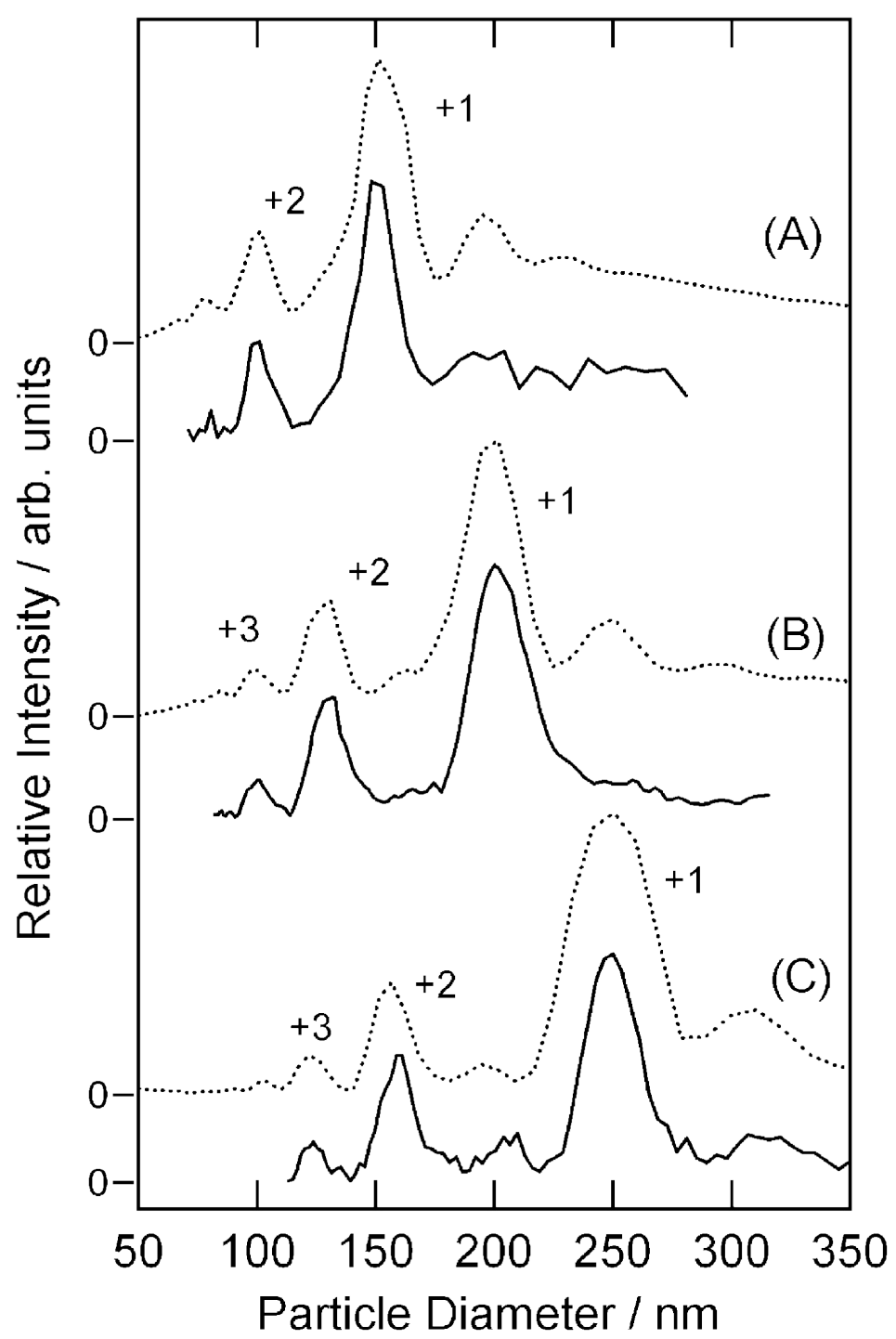

Fig. 1 Size distribution of variable size silica nanoparticles: (A) $150 \mathrm{~nm}$; (B) 200, and (C) $250 \mathrm{~nm}$. The size distributions are either probed by monochromatic synchrotron radiation ( $\mathrm{E}=85 \mathrm{eV}, \lambda=14.59 \mathrm{~nm}, 17^{\circ}$ scattering angle) in a nanoparticle beam (full curves) or by a condensation particle counter (dotted curves). 
proximately $10^{4}$, where the photon energy range between $80 \mathrm{eV}$ and $150 \mathrm{eV}$ is covered. This is suitable to excite the valence continuum below the Si 2p-edge as well as the Si 2pcontinuum. The size of the monochromatic synchrotron radiation in the scattering center depends on the size of the exit slit of the monochromator. Typical values are $17 \mu \mathrm{m} \times 60$ $\mu \mathrm{m}$ for large scattering angles $>6^{\circ}$ and $17 \times 6 \mu \mathrm{m}$ for $4^{\circ}-6^{\circ}$ scattering angles, where the slits are narrowed in order to avoid saturation of the detector due to strong forward scattering and the primary beam of synchrotron radiation.

The scattered radiation from free silica particles in a high vacuum surroundings is detected by a Csl-sensitized microchannel plate detector, which is mounted behind a variable size slit. We have used for the present experiments a slit width of $1.3 \mathrm{~mm}$, where the distance between the scattering center and the detector is $65 \mathrm{~mm}$. This yields an angular resolution of $1.15^{\circ}$. In this study, the detector is moved in the polarization plane of the linearly-polarized synchrotron radiation by using a stepping motor in the range of scattering angles between $4^{\circ}$ and $100^{\circ}$, where the precision of the angle is $\pm 0.1^{\circ}$. The absolute scattering angle is calibrated by the particle beam impinging on the detector, providing a distinct signal at $90^{\circ}$ scattering angle (Fig. 2), which is similar to previous work.[9] Note, that the detector can be mechanically adjusted to values between forward scattering, corresponding to $0^{\circ}$, and a $150^{\circ}$ scattering angle. This detector can be replaced by a total electron yield detector, which allows us to measure near-edge spectra of free nanoparticles.

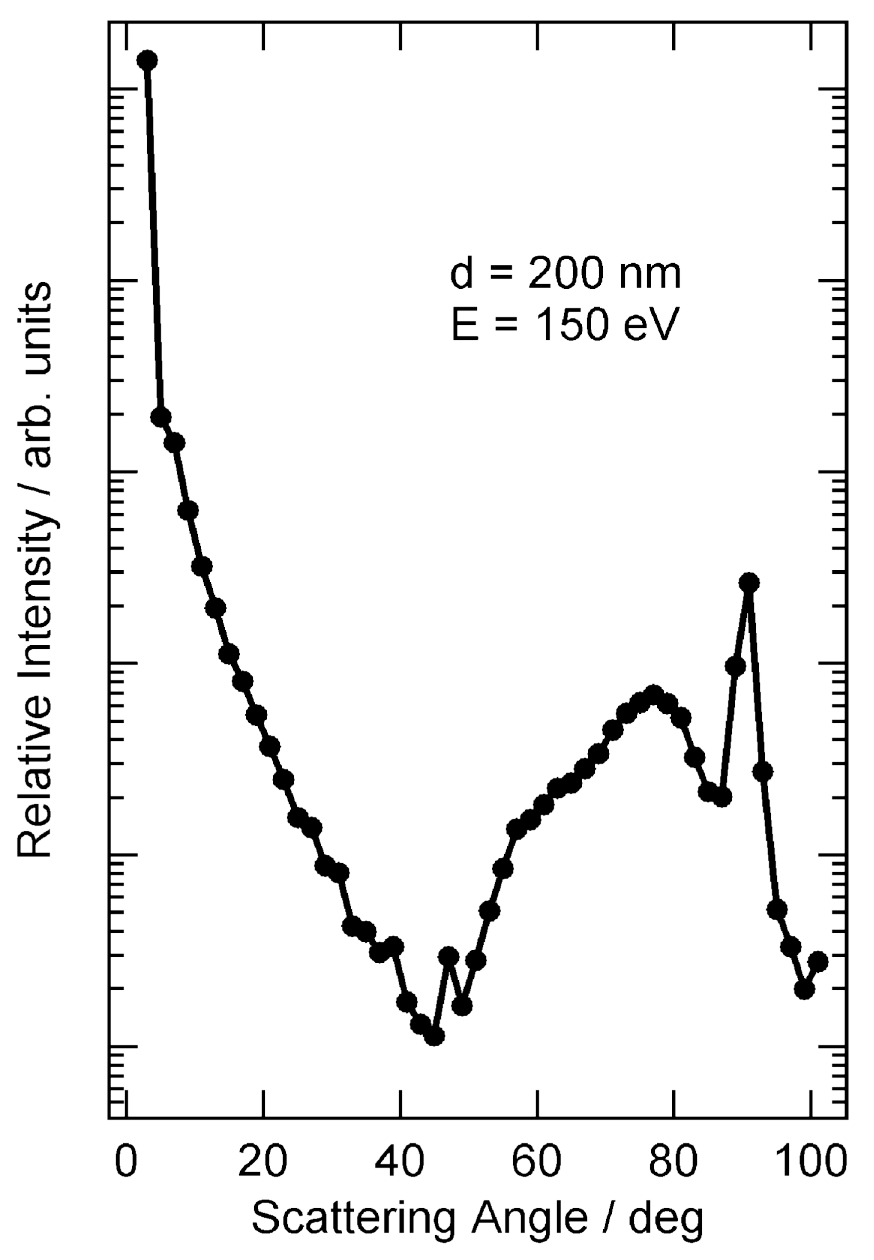

Fig. 2 Angle-resolved scattered light intensity in a logarithmic scale of 200 $\mathrm{nm}$ sized silica nanoparticles probed at $150.0 \mathrm{eV}$ photon energy. The sharp maximum at $90^{\circ}$ is a result of the particle beam hitting the detector. See text for further details. 
The experimental setup is used for the following experiments:

(i) Angle-resolved elastic light scattering provides specific information on the light scattering patterns in the regime of element-selective inner-shell excitation.

(ii) Spectroscopic experiments have been performed, where the photon detector is placed at a given scattering angle while scanning the photon energy near core level absorption edges.

The present work is focused on the Si $2 p$-regime, which is well-known from studies on macroscopic samples.[20,23,24]

\section{Results and discussion}

Fig. 1 shows a comparison of the particle size distribution recorded at different detection schemes: (i) use of a condensation particle counter (Fig. 1, dashed curves) and (ii) scattered light intensity of $E=85 \mathrm{eV}$ photons $(\lambda=14.59 \mathrm{~nm})$, as recorded after aerodynamic focusing, where the photon detector is placed at $17^{\circ}$ scattering angle (Fig. 1 , full curves). This photon energy is located below the Si $2 p$-edge. The scattering angle is well within the cone of forward scattering, as can be seen from Fig. 2, indicating that the scattered light intensity is preferentially due to elastic light scattering from particles. This is similar to previous work using VUV radiation.[9] Note that the dynamic range of the scattered light intensity covers about five orders of magnitude, as shown in Fig. 2 . This is about three orders of magnitude higher than in previous work.[9] The main difference compared to ref. 9 is that smaller scattering angles are accessible in the present setup. These contribute to a massive increase in scattered light intensity at low scattering angles. Furthermore, the Mie size parameter $x$ is substantially increased by a factor of $\sim 8$ in the present work, which is a result of shorter wavelength excitation $(8.2 \mathrm{~nm} \leq \lambda \leq 14.6 \mathrm{~nm}) \mathrm{com}$ pared to recent results.[]9 Specifically, in the case of particle sizes under investigation in this work, we derive $32 \leq x \leq 54$. Note that $x$ would be $<1.5$ in the visible regime, where most light scattering experiments are performed.

The size distributions shown in Fig. 1 probe particles as well as their aggregates. The present results indicate that the method of particle preparation is a sensitive parameter. The results obtained from the use of the condensation particle counter show that singly, doubly, and with minor intensity triply charged particles occur (dashed curves in Fig. 1). Besides isolated particles, one also observes aggregates of nanoparticles, which occur at larger sizes than the singly charged ones. The relative intensity of these aggregates is of the order of $20 \%$. This is substantially less than observed in previous work.[9] Aggregates of nanoparticles are formed if the particles already stick together in the liquid dispersion used to produce aerosol particles, or more than one particle is contained in a liquid aerosol droplet. The latter is easily avoided by diluting the suspension that contains the particles. The present results clearly indicate that the fraction of aggregates is substantially decreased if the scattered light intensity is used for probing the particle size. Evidently, this finding is a result of the transmission function of the aerodynamic lens, which is slightly different in design compared to earlier work.[9] The present suppression of aggregates is advantageous for the analysis of light scattering data, since the formation of aggregates is known to change the light scattering patterns.[9,17] Thus, such corrections are not needed in the present work, so that we may safely assume that we are preferentially studying isolated silica nanoparticles at low charge states. 


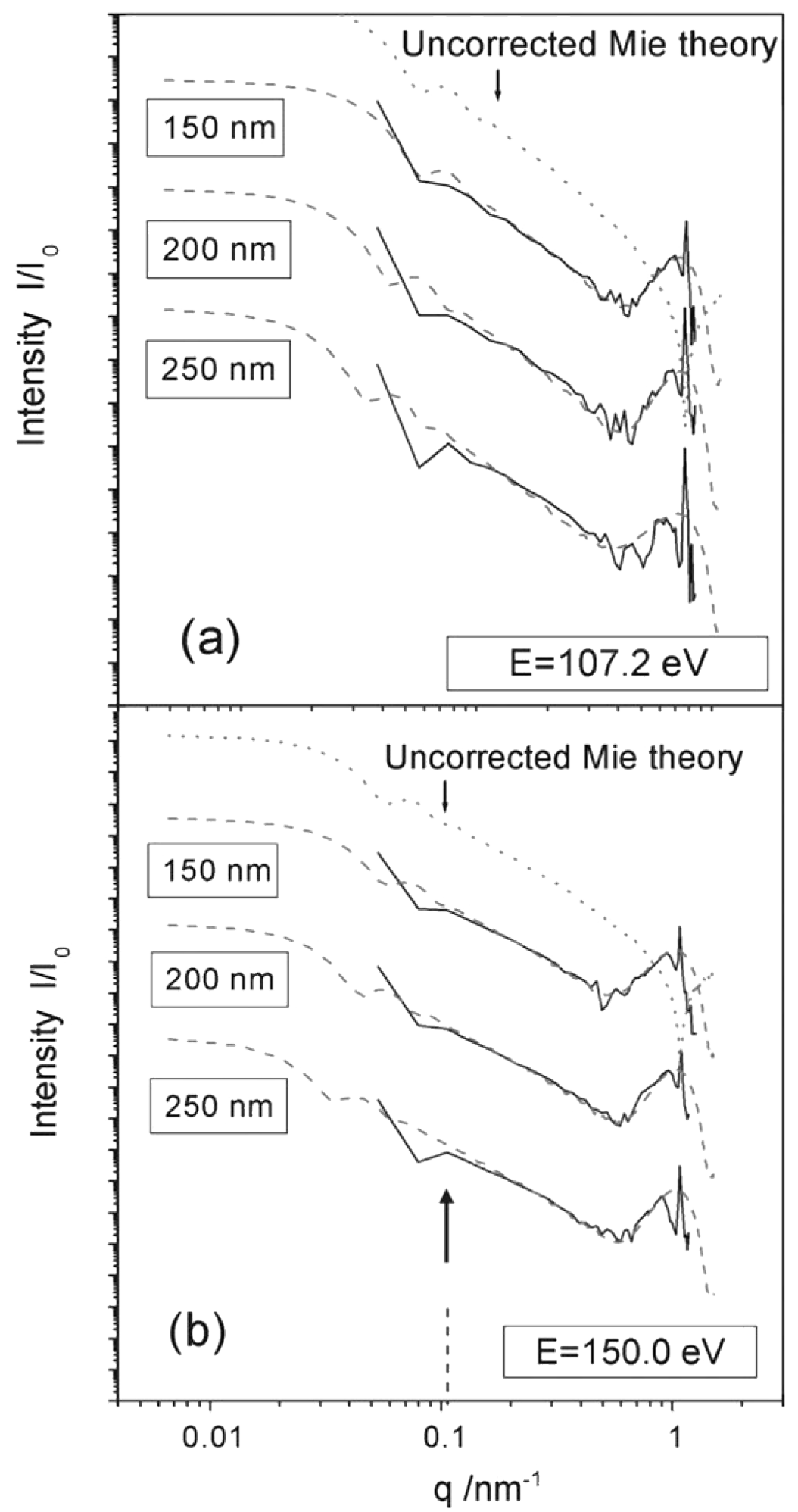

Fig. 3 Dependence of the normalized scattered light intensity from variable size silica nanoparticles at different photon energies $E$ as a function of the scattering wave vector q: (a) $E=107.2 \mathrm{eV}$, (b) $E=150.0 \mathrm{eV}$. The experimental data (solid lines) are compared to model results, as indicated by dashed curves (for further details: see text and eqn (3)). The results are compared to a Mie model for a perfectly smooth $250 \mathrm{~nm} \mathrm{SiO} 2$ particle (dotted curve at the top of each plot). The position corresponding to the total external reflection angle is indicated by an upward arrow. The curves are vertically displaced in order to visualize changes in shape. 
The raw data of the scattered light intensity are shown in Fig. 2, where highest intensity is observed at low scattering angles. Alternatively, the scattered light intensity can be plotted as a function of the scattering wave vector $q$, as shown in Fig. 3 . The scattering wave vector is given by $q=4 \pi \lambda^{-1} \sin (\theta / 2)$, where $\lambda$ is the wavelength of the radiation and $\theta$ is the scattering angle. This presentation of the experimental results is similar to earlier work of Sorensen and Fischbach, 11 who have used the dimensionless product $q R$.

Fig. 3 presents the experimental results along with fits of the $q$-dependences of the normalized scattered light intensity of differently sized $\mathrm{SiO}_{2}$ particles recorded at $107.2 \mathrm{eV}$ and $150.0 \mathrm{eV}$ photon energy, respectively. The lower photon energy is located in the Si $2 p-$ near-edge regime, whereas the higher one corresponds to the $\mathrm{Si} 2 \mathrm{p}$-continuum. The dashed curves result from calculated results, where the transmitted part of scattered radiation is calculated in the framework of Mie theory by using the program package MiePlot.[2,6,9] This requires to use proper values of the refraction and absorption coefficients, as obtained from reflectivity measurements on thin $\mathrm{SiO}_{2}$ films.[24] A comparison of the experimental results with a simulation that considers exclusively Mie scattering (see dotted curve at the top of each plot shown in Fig. 3) indicates that there are distinct differences as a function of $q$. We conclude from this, that Mie-scattering cannot be exclusively used to model the experimental results over the entire range of $q$. This is specifically evidenced by a distinct minimum, which occurs near $q \approx 0.43 \mathrm{~nm}^{-1}$ at $E=107.2 \mathrm{eV}$ and near $q \approx 0.55 \mathrm{~nm}^{-1}$ at $150.0 \mathrm{eV}$ in the experimental curves, as shown in Fig. 3.

Mie theory predicts that the distinct minimum should occur at a scattering angle of $90^{\circ}$, which corresponds to $q \approx 0.77 \mathrm{~nm}^{-1}$ at $107.2 \mathrm{eV}$ and $q \approx 1.07 \mathrm{~nm}^{-1}$ at $150 \mathrm{eV}$, respectively (cf. dotted curves in Fig. 3). This is unlike the experimental results. At a first glance one might assign this discrepancy to a strong increase in the phase shift parameter $\rho$, leading to a violation of the Rayleigh-Debye-Gans (RDG) theory, which requires that $\rho<1$ and In $-1 \mid<1$, respectively.[2] The phase shift parameter $\rho$ is expressed by $\rho=2 k_{0} R|n-1|$, (see ref. [11] for details), where $k_{0}=2 \pi / \lambda$ is the wave vector of the incident wave, $R$ is the particle radius, and $n$ is the refractive index of the sphere. In the present case, using e.g., $E=$ $150 \mathrm{eV}, R=125 \mathrm{~nm}$, and $n \approx 0.98$, we derive the phase shift value of $\rho=7.6$, which brings us to the intermediate regime between the applicability of RDG theory and anomalous diffraction.

Thus, one possibility to rationalize the deviation of the first, strong Mie minimum in the experimental scattered light intensity as a function of $q$, shown in Fig. 3, could be found in an unexpected strong change in refractive index $n$ in the surface region. A possible origin of such a change might be found either as a result of surface contamination or a strong structural change in the surface layer. However, attempts to fit the $q$-dependence of Mie scattering to the simple model of a coated sphere, by considering either surface contaminations or a rough surface of coated spheres, failed. This is because one obtains from such fits unrealistically high values for the index of refraction, which range between 1.7 and 2.5. Therefore, we discount this assignment. Further, additional fits to the experimental results using pure Mie theory by varying the index of refraction of the spheres, which are different from published values,[24] also failed. They give similarly large values of $n \gg$ 1 , which is unlike the published values ranging between 0.97 and 0.99 in the energy regime under investigation.[24]

A meaningful and correct fit of the experimental results is only achieved by considering strong reflectivity attenuation of the incoming beam from a rough surface of $\mathrm{SiO}_{2}$, as will be outlined below. As a result, the expression for the detected scattered light intensity contains two parts, one coming from the Mie scattering from an ideal sphere and the other one 


\section{Detector}

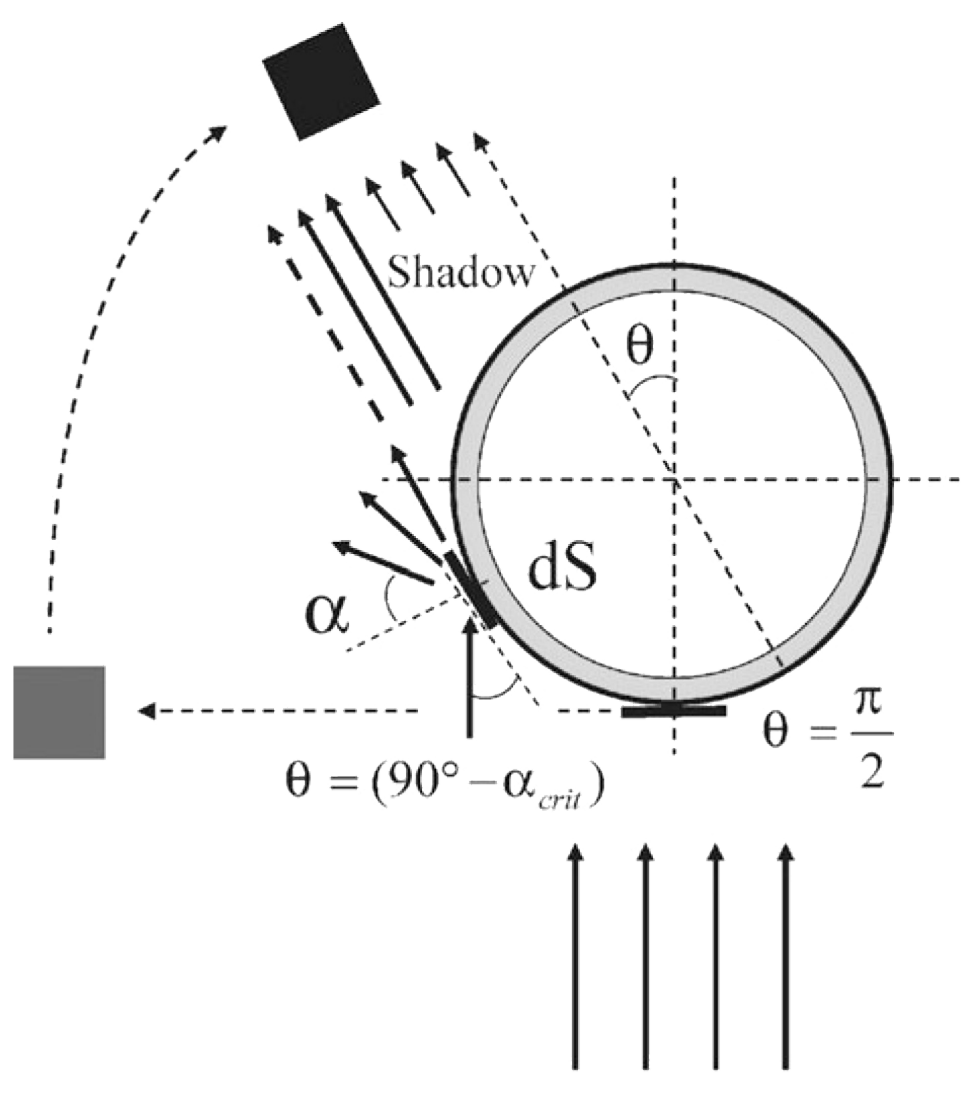

\section{Incident beam}

Fig. 4 Schematic presentation of the scattering geometry. The edge of the diffusely scattered beam reaching the detector at the scattering angle $\theta$ is indicated by a coarsely dashed arrow.

is due to changes in reflectivity caused by a rough and graded surface, yielding $I_{\text {res }}=\|_{\text {ideal }}$ scat $+\Delta l_{\text {refl. }}$. The scattered part for perfect spheres ${ }^{\prime}$ deal ${ }_{\text {scat }}$ results from Mie calculations using the tabulated values of the highly dispersive extinction coefficient, corresponding to $n$ and $k$ (see ref. 24) and by taking into account the experimental particle size distribution, which is derived from the present experiments (see below). The change in the reflective part is modelled by a combination of diffuse $R_{\text {diff }}$ and specular reflectivity $R_{\text {spec. }}$. The relative intensity of reflected radiation $I=R_{\text {diff }} /\left(R_{\text {diff }}+R_{\text {spec }}\right)$ is corrected for the attenuation due to the experimentally estimated surface roughness $\sigma$. We use for $\sigma$ typical experimental values, ranging between 1 and $2 \mathrm{~nm}$,[24] which is added to the $\sim 20 \mathrm{~nm}$ thickness of a radial density gradient layer. Such a layer may, at least in part, result from residual solvent on the particles. This appears to be plausibe, as the particles are transferred within 5 to $10 \mathrm{~s}$ from the liquid phase into the high vacuum chamber, where the scattering process occurs. Therefore, it is likely that some solvent remains on the surface. Such a change in reflected light is treated in the framework of geometrical optics, since anomalous diffraction can still be ignored at $\rho=7.6$.[28] The consideration of diffuse reflection is applicable to the present case, since particle diameters in the range of $150-250 \mathrm{~nm}$ are much larger than the wavelength, which is of the order of $8-15 \mathrm{~nm}$.

According to van de Hulst,[28] the scattering patterns of small spheres with elements obeying the Lambert law of diffuse reflection can be calculated by using the theory of 
Schoenberg.[29] We show in Fig. 4 the geometry of diffuse reflection, which is discussed below in greater detail.

The experimentally detected phase shift observed in Fig. 3 is related to the phase shift across the spherical particle in Mie theory $\left(\rho=2 k_{0} R I n-1 /\right)$,[11] as mentioned above. We have included the anisotropy of the scattering angle $\theta$ in the Gaussian power spectral density function of the surface roughness by using the correlation function $g(k)$ :

$$
g(k) \sim \pi \sigma^{2}(2 R)^{2} \exp \left(-\left[2 k_{0} R|n-1| \cos \theta\right]^{2}\right)=\sigma^{2} \exp \left(-[\rho \cos \theta]^{2}\right)
$$

Here, $2 R|n-1| \cos \theta$ is the optical path difference through the sphere along $\theta$ (cf. ref. 28 and 30 ) and the correlation length is taken to be $2 R$, corresponding to the particle diameter. Note that eqn (1) represents an empirical approximation of the exact solution for calculating the reflectivity of a rough surface using the values mentioned above. This also requires the use of results from ref. 30 , where the integration over the illuminated surface of a spherical $\mathrm{SiO}_{2}$ particle has to be taken into account.

In the schematic presentation shown in Fig. 4, a surface element dS reflects the flux $\cos (a) d S / \pi$ per unit solid angle in the direction of a given angle a relative to the surface normal. By integrating over all angles up to the shadow and using the attenuation weight given by eqn (1), we estimate the contribution of the diffusely scattered light:

$$
R_{\mathrm{diff}} \sim \pi \sigma^{2}(2 R)^{2} \exp \left(-[\rho \cos \theta]^{2}\right)[\sin \theta+(\pi-\theta) \cos \theta]
$$

The contribution of specular reflectivity $R_{\mathrm{spec}}$ is considered for a comparison by the Fresnel formula for parallel polarized light[31]

$$
R_{\mathrm{spec}}=\left|\frac{\epsilon \cos \alpha-\left(\epsilon-\sin ^{2} \alpha\right)^{1 / 2}}{\epsilon \cos \alpha-\left(\epsilon+\sin ^{2} \alpha\right)^{1 / 2}}\right|^{2}
$$

where the notation of ref. 24 is used with $\varepsilon=1-\varepsilon_{1}+i \varepsilon_{2}=(n+i k)^{2} . \varepsilon_{1}$ and $\varepsilon_{2}$ correspond to the real and imaginary parts of the dielectric constant, respectively. Further, $n$ and $k$ represent the real and imaginary parts of refractive index, and $a$ is the angle relative to the normal. In general, it is not possible to distinguish quantitatively between the contributions from specular and diffuse reflection, simply by fitting the experimental curves. However, one important observation in the region of small values of $q$ is deduced from the curve fits, as shown in Fig. 3 : there is a clear step visible near $q \approx 0.1 \mathrm{~nm}^{-1}$. Specifically, this feature occurs at $q=0.12 \mathrm{~nm}^{-1}$ in the case of $250 \mathrm{~nm}$ particles studied at $E=150 \mathrm{eV}$ (see arrow in Fig. $3(b)$ ). This feature is quite close to the calculated value of $a_{c r i t}=80.8^{\circ}$ in total external reflection using the realistic value of $n_{\mathrm{SiO}_{2}}=0.987$. Thus, we assign this distinct step in the experimental results to total external reflection. Note, that the expression $\varepsilon$ $-\sin ^{2} \mathrm{a}$ in the numerator of $R_{\text {spec }}$ in eqn (3) is mainly governed by absorption at $\mathrm{a}=\mathrm{a}_{\mathrm{crit}}$. 
This correlates well with the step in the experimental curves, as indicated by the arrow in Fig. 3(b). One can also see from Fig. 3 that all experimental curves are successfully reproduced by the model outlined above reaching down to small glancing angles, which correspond to small values of $q$. These are close to the critical angle $a_{\text {crit. Interestingly, there is }}$ no such feature modelled for the pure Mie scattering part in this regime of $q$. However, there is a similar feature that occurs as a low amplitude wiggle in the Mie scattering at somewhat smaller $q$ (see Fig. 3). The absence of the experimentally observed shoulder in Fig. 3 in the theoretical curve at low $q$ values $\left(q \sim 0.1 \mathrm{~nm}^{-1}\right)$ is evidently related to TER, according to Fresnel formalism shown in eqn (3). The occurrence of this feature at lower values of $q$ compared to the experimental results is likely a result of limitations of the present model, which may be improved by using the approach outlined in ref. 30 .

The expected values for $2 R|n-1|$ for the given photon energies and particle sizes are ranging between 1.7 and 3.5. Consistently, the values extracted from the fit to the experimental data shown in Fig. 3 are observed in the value range between 1.9 and 3.0. This agreement indicates that the only adjustable parameter in our model is the fraction of the incoherently reflected light, which is due to the surface roughness and radial density gradient of residual solvent, as discussed above. Note, that the exact modelling of the theory outlined in ref. 30 would even make this single fit parameter superfluous. Similarly, there are small but detectable differences in particle size-dependent phase shifts. These are found to be smaller than expected from the definition of the phase shift $\rho$. Evidently, the deviations from RDG theory, which are solely a function of $q R$, are not too strong. At the same time the size of the particles does not affect their roughness, indicating that the roughness remains a constant quantity.

The position of the broad minimum mentioned above evidently depends on the particle radius. Fig. 3(a) and (b) indicate that the broad minimum is well modelled by the present approach and the position of this minimum is shifted by a factor of $\sim 1.5$ to larger values of $q$ when changing the particle size from $D=150 \mathrm{~nm}$ to $250 \mathrm{~nm}$. This difference in energydependent shift of the minimum is at a first glance somewhat unexpected, since $q$ scales with the photon energy $E$ and will lead to a constant value of the minimum in the scattering curves as a function of $q$. A plausible reason for this discrepancy with the experimental results is found in the energy dependence of the index of refraction. Specifically, one derives from ref. 24 that $|n-1|=0.011$ at $E=107.2 \mathrm{eV}$ and $|n-1|=0.014$ at $E=150.0 \mathrm{eV}$, respectively. The position of the minimum scales with the product $I n-1 \mid \cos \theta$, which indicates that this feature should be found at larger values of $q$, when the photon energy is increased. This is in full agreement with the experimental results shown in Fig. 3.

Another presentation of the experimental results, that has been suggested before,[11] is to plot the scattered light intensity over the dimensionless parameter $q R$. This yields power laws, as shown in Fig. 5, where the slopes are indicative for the properties of the scattering process. One expects to obtain from this approach for systems, which slightly deviate from RDG theory, slopes of $(q R)^{-2}$.[11] These have to be followed by a $(q R)^{-4}$ Porod law at higher $q R$ values.[11] We show in Fig. 5, as one typical example, such a plot, which is recorded at $E=150 \mathrm{eV}$ using $250 \mathrm{~nm}$ silica particles. We show also for comparison in Fig. 5 results from Mie simulations of a sphere (dotted curve in Fig. 5), yielding a $(q R)^{-2}$ dependence. The same slope can also be rationalized by connecting neighboring Mie maxima, as indicated by the dashed line in Fig. 5 . There is, however, a distinct $(q R)^{-4}$ dependence in the present results shown in Fig. 5. This is assigned to the occurrence of a gradient of a smoothly decreasing scattering intensity of $\sim 30 \mathrm{~nm}$ thickness in the surface region of the particles, similar to the discussion given in ref. 22). 


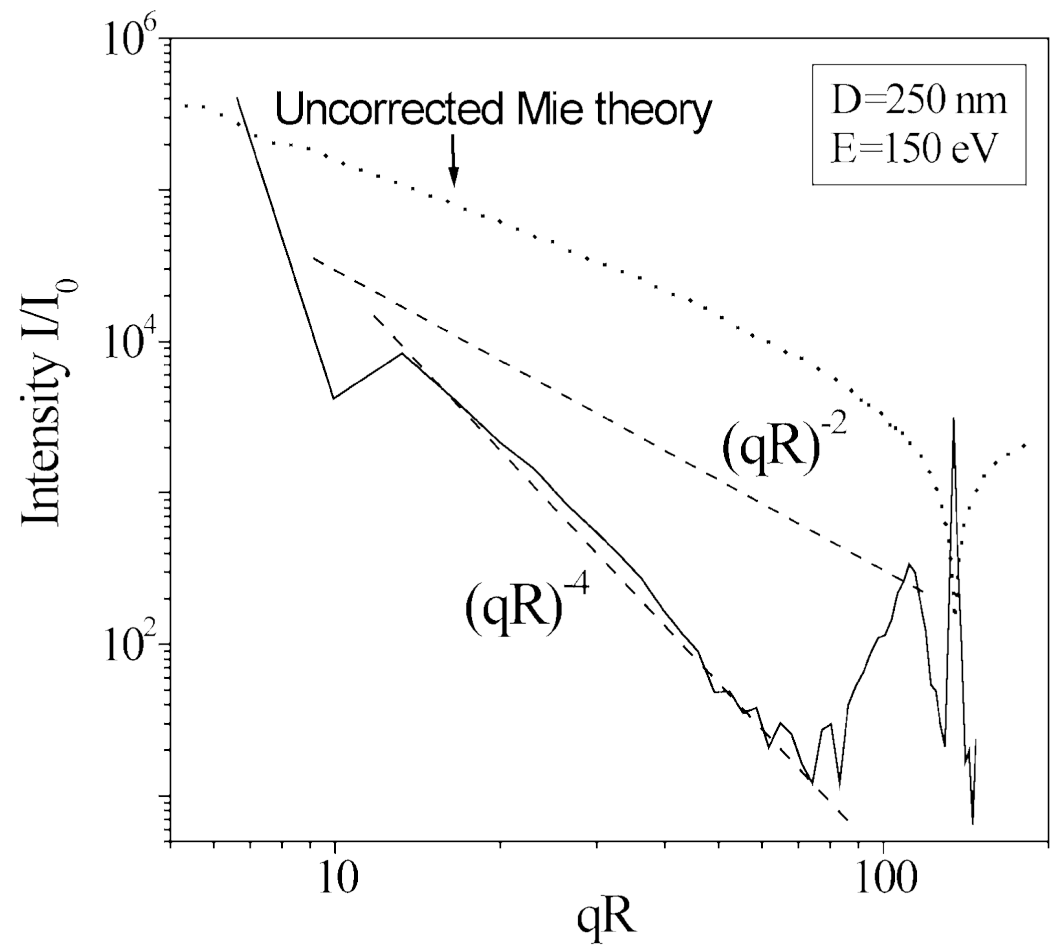

Fig. 5 Scattered light intensity from $250 \mathrm{~nm} \mathrm{SiO}$ particles at $150.0 \mathrm{eV}$ photon energy as a function of the dimensionless parameter $q R$ (full curve). Slopes corresponding to $(q R)^{-2}$ and $(q R)^{-4}$ are indicated by dashed lines. The dotted line is obtained from a Mie model of a perfect sphere.

Expulsion of the electromagnetic field to the boundary of spherical particles is another important aspect to be considered.[32] In the photon energy range of this study (85-150 $\mathrm{eV}$ ), in which the incoming photon wavelength $\lambda$ is by about one order of magnitude smaller than the particle size, the electromagnetic field is predominantly localized in the region close to the surface. The thickness of this surface layer accounts for $\sim 20 \%$ of the particle radius.[32] This implies that contributions from the particle core to the elastically scattered light intensity can be neglected. As a result, the relative importance of the surface contribution and the sensitivity to surface properties of free nanoparticles probed by the reflected light is significantly enhanced in the soft X-ray regime. This supports our conclusion that the $(q R)^{-4}$ regime shown in Fig. 5 comes from the density gradient of the surface layer, which is most likely dominated by residual solvent.

The $\mathrm{Si} 2 \mathrm{p}$ near-edge regime of various $\mathrm{SiO}_{2}$ polymorphs as well as amorphous $\mathrm{SiO}_{2}$ have been studied before.[23] The features occurring in the near-edge regime (NEXAFS) of $150 \mathrm{~nm}$ silica nanoparticles are shown in Fig. 6, which are in agreement with earlier results. [20,23,24] Details on the spectral assignments of the near-edge features can be found in these previous works. Additional experiments indicate that the near-edge spectra of differently-sized silica particles do not show any particle size dependence. This is quite expected, since the local surrounding near the absorbing Si sites is probed, which evidently does not change in the regime of nanoparticles. Fig. 6 also shows the scattered light intensity in the Si $2 p$-regime recorded at different scattering angles. The photon energy scale and the spectral shape of both reflection and absorption appears to be in general agreement with previous results.[20,23] 


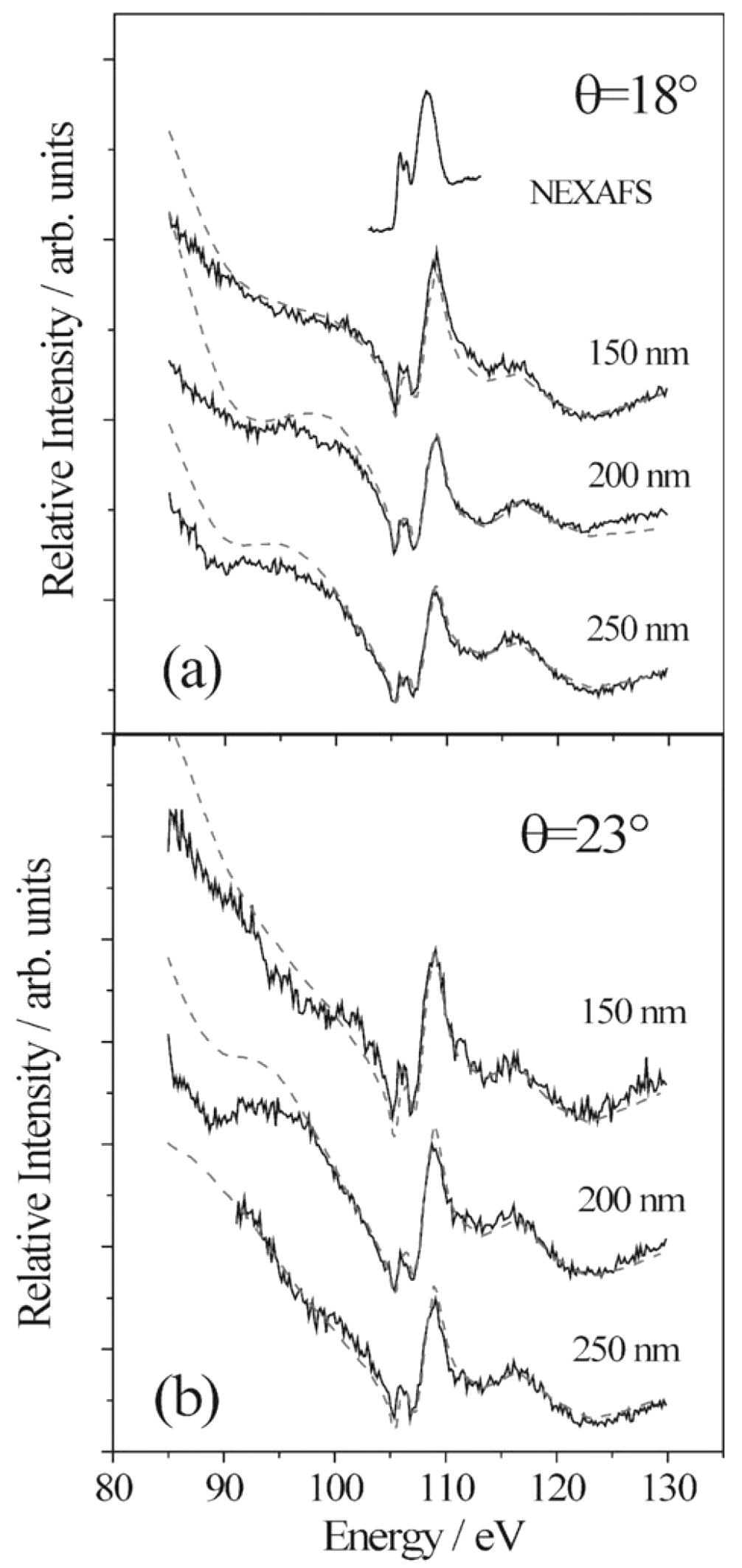

Fig. 6 Dependence of the normalized scattered light intensity from variable size silica particles on the incident photon energy $E$ in the Si $2 p$ regime (full curves): (a) $18^{\circ}$ scattering angle, (b) $23^{\circ}$ scattering angle. The dashed curves correspond to the modelled spectra using a pure Mie model including the size distributions compiled in Table 1 along with the optical constants taken from ref. [24]. The near-edge spectrum (NEXAFS) at the top of the Figure is obtained from total electron yield measurements of $150 \mathrm{~nm}$ silica particles. 
Simulations of the energy scans of the scattered light intensity are also shown in Fig. 6 (dashed lines), where a pure Mie model[2,6] is used. The applicability of this approach is justified for the regime of large scattering angles $\theta \geq 18^{\circ}$. This is because the Mie model yields reasonable agreement with the experimental results at sufficiently large $q$-values, which are above the distinct step, that is discussed above in terms of TER, and below the broad minimum (cf. Fig. 3). The fine structure in the $100-125 \mathrm{eV}$ regime, corresponding to the highly dispersive part of the complex refraction index, taken from ref. [24], is well reproduced by the present model results on variable-size nanoparticles. However, the results shown in Fig. 6 indicate that there are significant deviations from the Mie model, especially below $90 \mathrm{eV}$. These deviations from the Mie simulations to experimental spectra can be either attributed to diffuse scattering from a surface layer of the remaining solvent or to the lack of further corrections given in eqn (2), as well as previous work.[30]

Fitting the experimental energy scans in the Si $2 p$-regime to Mie theory is found to be sensitive to the $\mathrm{SiO}_{2}$ particle size distribution in the nanoparticle beam (cf. dashed curves in Fig. 6). This is similar to recent results recorded in the valence regime, where it is found that Mie oscillations smear out with increasing the size distribution.[9] Fig. 7 clearly shows how sensitive the size distribution changes the photon energy dependencies, by using different log-normal size distributions for the $D=250 \mathrm{~nm}$ sample. This represents an arbitrarily chosen example, where the scattered light is detected at $\theta=23^{\circ}$. Best agreement is observed for these conditions at $4.6 \%$ size distribution (Table 1), whereas monodisperse particles $(0 \%$ size distribution) also show distinct oscillations in the pre-edge regime. These are evidently not observed in the experimental results. Such oscillations smear out with increasing size distribution and the narrow features near $105 \mathrm{eV}$ start to sharpen up, as is observed in the experimental spectrum (cf. Fig. 6). Increasing the width of the size

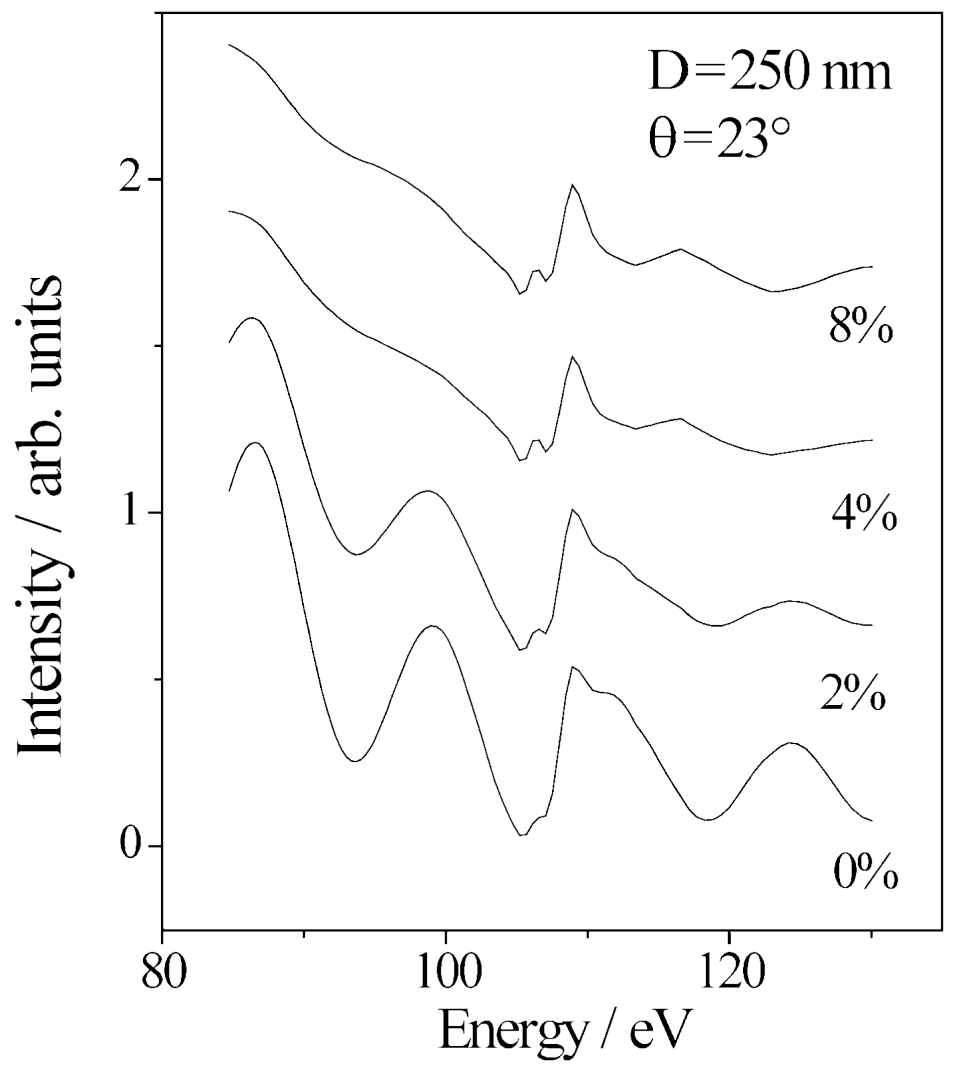

Fig. 7 Mie simulations for a $\mathrm{SiO}_{2}$ sphere $\left(D=250 \mathrm{~nm}\right.$ at $23^{\circ}$ scattering angle $\theta$ ) in the $\mathrm{Si} 2 p$-regime of variable size log-normal distributions, as indicated. The size distribution of $0 \%$ corresponds to monodisperse particles. 
distribution to larger values beyond the best fit to the experimental results indicates that there are less significant changes than those observed for narrow size distributions $(<4 \%)$. There are, however, characteristic changes in amplitudes of the spectral features, as is evident from a comparison between a $4 \%$ and an $8 \%$ distribution, indicating that an $8 \%$ size distribution is too wide. A comparison with previous work on amorphous $\mathrm{SiO}_{2}$ [24] indicates that the clearly split feature near $105 \mathrm{eV}$, as shown in Fig. 6, also occurs. It is similar in shape as observed from the size distributions, whereas the monodisperse particles indicate no such splitting, as observed from Mie simulations (cf. Fig. 7, bottom trace). Clearly, this similarity in spectral shape indicates that these are not unique. The major difference between the particle size distributions shown in Fig. 6 and the amorphous films (cf. ref. [20]) is the significantly different scattering angle: a small scattering angle of $\sim 0.6^{\circ}$ from the solid yields a similar spectral signature to the present particle distributions at considerably larger scattering angles.

Table 1 Log-normal particle size distribution as obtained from a fit to the Mie model from the energy resolved experiments shown in Fig. 6

\begin{tabular}{|c|c|c|}
\hline Nominal diameter D/nm & Center of size distribution/nm & Width of size distribution (in \%) \\
\hline 150 & 147 & 71 \\
\hline 200 & 192 & 50 \\
\hline 250 & 240 & 46 \\
\hline
\end{tabular}

The resulting log-normal distributions of the particle sizes that are used to fit the results shown in Fig. 6 are compiled in Table 1. These are consistent with the TEM and DMA results discussed above, indicating that size distributions can be sensitively and reliably derived from the scattered light intensity in the near-edge regime. The data shown in Table 1 also indicate that the relative widths of the distributions decrease as a function of particle size, which is consistent with earlier work[9] and results from electron microscopy.[26]

\section{Conclusions}

It is shown that properties of isolated nanoparticles are sensitively probed in the soft Xray regime. This is accomplished by studying a free nanoparticle beam containing variable size silica particles in the Si 2p-regime (85-150 eV). Angle-resolved light scattering provides detailed information on the scattering mechanisms, which contribute to the innershell excitation regime. The present results indicate that Mie scattering cannot fully explain the angle-resolved scattered light intensity. A simple model is developed, which considers the surface roughness, total external reflection, and specular reflection. It allows us to fit the experimental results and to rationalize distinct deviations from Mie theory. The present results clearly show evidence for the surface sensitivity of the present approach.

Energy scans near the Si 2p-edge at a fixed scattering angle show size-effects of the scattered light intensity. This provides a sensitive measure for both the size as well as the size distribution of free nanoparticles. The results are consistent with other approaches of particle size determination.

The present results indicate that inner-shell excitation has the potential to provide a sensitive probe for size effects in nanoscopic matter. Specifically, it is anticipated that sur- 
face properties of free variable size and surface functionalized nanoparticles can be selectively probed by element-specific core level excitation and elastic light scattering, which is the subject of ongoing work.

\section{Acknowledgements}

Financial support by the BMBF (contract $05 \mathrm{KS} 4 \mathrm{WW1/7}$ ) and the Fonds der Chemischen Industrie is gratefully acknowledged.

\section{References}

[1] M. Kerker, The Scattering of Light and Other Electromagnetic Radiation, Academic Press, New York, 1969.

[2] C. F. Bohren and D. R. Huffman, Absorption and Scattering of Light by Small Particles, Wiley, New York, 1983.

[3] R. Finsy, Adv. Colloid Interface Sci., 1994, 52, 79.

[4] Aerosol Measurement, ed. P. A. Baron and K. Willeke, Wiley, New York, 2001.

[5] G. Mie, Ann. Phys. (Leipzig), 1908, 25, 377.

[6] P. Laven, Appl. Opt., 2003, 42, 436.

[7] J. Shu, K. R. Wilson, A. N. Arrowsmith, M. Ahmed and S. R. Leone, Nano Lett., 2005, 5, 1009.

[8] M. B. Huglin, Light Scattering from Polymer Solutions, Academic Press, London, 1972.

[9] J. N. Shu, K. R. Wilson, M. Ahmed, S. R. Leone, C. Graf and E. Rühl, J. Chem. Phys., 2006, 124, 034707.

[10] J. N. Shu, K. R. Wilson, M. Ahmed and S. R. Leone, Rev. Sci. Instrum., 2006, 77, 043106.

[11] C. M. Sorensen and D. J. Fischbach, Opt. Commun., 2000, 173, 145.

[12] M. Xu and R. R. Alfano, Opt. Commun., 2003, 226, 1.

[13] J. C. Jonsson, G. B. Smith and G. A. Niklasson, Opt. Commun., 2004, $240,9$.

[14] (a) K. S. Harnard, R. Roth, J. Rockenberger, T. van Buuren and A. P. Alivisatos, Phys. Rev. Lett., 1999, 83, 3474; (b) A. S. Ethiraj, N. Hebalkar, S. K. Kulkarni, R. Pasricha, C. Dem, M. Schmitt, W. Kiefer, L. Weinhardt, S. Joshi, R. Fink, C. Heske, C. Kumpf and E. Umbach, J. Chem. Phys., 2003, 118, 8945.

[15] U. Pietsch, Phys. Rev. B, 2002, 66, 155430.

[16] T. Araki, H. Ade, J. M. Stubbs, D. C. Sundberg, G. E. Mitchell and A. L. D. Kilcoyne, Appl. Phys. Lett., 2006, 89, 124106.

[17] (a) M. Quinten and U. Kreibig, Surf. Sci., 1986, 172, 557; (b) M. Quinten and U. Kreibig, Appl. Opt., 1993, 32, 6173.

[18] M. Grimm, B. Langer, S. Schlemmer, T. Lischke, U. Becker, W. Widdra, D. Gerlich, R. Flesch and E. Rühl, Phys. Rev. Lett., 2006, 96, 066801.

[19] H. Döllefeld, C. McGinley, S. Almousalami, T. Möller, H. Weller and A. Eychmüller, J. Chem. Phys., 2002, 117, 8953.

[20] O. A. Ershov, D. A. Goganov and A. P. Lukirskii, Sov. Phys. Sol. State, 1966, 7, 1903. 
[21] A. V. Vinogradov, N. N. Zorev, I. V. Kozhevnikov, S. I. Sagitov and A. G. Tur' ynskii, Sov. Phys. JETP, 1988, 67, 1631.

[22] S. K. Sinha, E. B. Sirota, S. Garoff and H. B. Stanley, Phys. Rev. B, 1988, 38, 2297.

[23] D. Li, G. M. Bancroft, M. Kasrai, M. E. Fleet, R. A. Secco, X. H. Feng, K. H. Tan and B. X. Yang, Am. Mineral., 1994, 79, 622.

[24] E. Filatova, V. Lukjanov, R. Barchewitz, J. M. André, M. Idir and Ph. Stemmler, J. Phys.: Condens. Matter, 1999, 11, 3355.

[25] W. Stöber, A. Fink and E. J. Bohn, J. Colloid Interface Sci., 1968, 26, 62.

[26] A. van Blaaderen, J. van Geest and A. Vrij, J. Colloid Interface Sci., 1992, 154, 481.

[27] (a) P. Liu, P. J. Ziemann, D. B. Kittelson and P. H. McMurry, Aerosol Sci. Technol., 1995, 22, 293; (b) P. Liu, P. J. Ziemann, D. B. Kittelson and P. H. McMurry, Aerosol Sci. Technol., 1995, 22, 315.

[28] H. C. van de Hulst, Light Scattering by Small Particles, Dover, New York, 1981.

[29] E. Schoenberg, Grundlagen der Photometrie, in Theoretische Astrophysik, Springer, Berlin, 1929, p. 64.

[30] J. M. Elson, J. P. Rahn and J. M. Bennet, Appl. Opt., 1983, 22, 3207 [Links].

[31] F. Stern, in Solid State Physics, ed. F. Seitz and D. Turnbull, Academic, London, 1963, vol. 15, p. 320.

[32] D. Q. Chowdhury, P. W. Barber and S. C. Hill, Appl. Opt., 1992, 31, 3518. 